This is the peer reviewed version of the following article: Felisberti, Fatima and Terry, Philip (2015) The effects of alcohol on the recognition of facial expressions and microexpressions of emotion : enhanced recognition of disgust and contempt. Human Psychopharmacology: Clinical and Experimental, $30(5), \mathrm{pp}$. 384-392., which has been published in final form at http://dx.doi.org/10.1002/ hup.2488. This article may be used for non-commercial purposes in accordance with Wiley Terms and Conditions for Self-Archiving. 


\section{The effects of alcohol on the recognition of facial expressions and microexpressions of emotion: enhanced recognition of disgust and contempt}

Fatima Felisberti and Philip Terry

Department of Psychology, Kingston University, Penrhyn Road, Kingston-upon-Thames, Surrey KT1 2EE, United Kingdom

Correspondence to Philip Terry at: Department of Psychology, Kingston University, Penrhyn Road, Kingston-upon-Thames, Surrey KT1 2EE, UK. Tel: 020-8417-7161

Email: p.terry@kingston.ac.uk.

Conflicts of Interest: None

Running Title: Alcohol and microexpressions of emotion

Keywords: Alcohol; Disgust; Facial expressions of emotion; Microexpressions; Emotion

Acknowledgements: The authors would like to thank Les Spaine for research assistance. The study was supported by funds from the Faculty of Arts and Social Sciences at Kingston University. 


\section{ABSTRACT}

Objective: The study compared alcohol's effects on the recognition of briefly-displayed facial expressions of emotion (so-called "microexpressions") with expressions presented for a longer period of time. Method: Using a repeated-measures design, 18 participants were tested three times (counterbalanced), after: (1) a placebo drink; (2) a low-to-moderate dose of alcohol (0.17 g/kg women; $0.20 \mathrm{~g} / \mathrm{kg}$ men); and (3) a moderate-to-high dose of alcohol $(0.52$ $\mathrm{g} / \mathrm{kg}$ women; $0.60 \mathrm{~g} / \mathrm{kg}$ men). On each session, participants were presented with stimuli representing six emotions (happiness, sadness, anger, fear, disgust, contempt) overlaid on a generic avatar in a 6-alternative forced-choice paradigm. A neutral expression $(1 \mathrm{~s})$ preceded and followed a target expression presented for $200 \mathrm{~ms}$ (microexpressions) or $400 \mathrm{~ms}$. Participants mouse-clicked the correct answer. Results: The recognition of disgust was significantly better after the high dose of alcohol than after the low dose or placebo drinks at both durations of stimulus presentation. A similar profile of effects was found for the recognition of contempt. There were no effects on response latencies. Conclusion: Alcohol can increase sensitivity to expressions of disgust and contempt. Such effects are not dependent on stimulus duration up to $400 \mathrm{~ms}$ and may reflect contextual modulation of alcohol's effects on emotion recognition. 


\section{INTRODUCTION}

It is widely acknowledged that alcohol administered at social doses can alter a person's emotional state, either directly or indirectly via its effects on cognition (e.g. Cooper et al., 1995; Curtin et al., 2001; Donohue et al., 2007; Westmaas et al., 2007; Sayette et al., 2012). Indeed, many problems arising from alcohol intoxication have been attributed to the drug's effects on emotion regulation (e.g. Giancola 2000, 2004; Eckhardt, 2007; Williams and Hasking, 2010; Stappenbeck and Fromme, 2014). However, it is now increasingly apparent that alcohol consumption may also impact on a person's capacity to interpret the emotional states of other people. Facial expressions are key indicators signalling a person's emotional state, and the accurate recognition of such expressions is critical for a successful navigation of the social environment. Therefore if alcohol impairs the recognition of emotional expressions then it might lead to adverse consequences; in particular, it has been suggested that misinterpreting facial expressions in a social context may underpin some alcohol-related aggression (Borrill et al., 1987; Blair, 2003; Attwood et al., 2009a; Attwood et al., 2009b, Craig et al., 2009; Kamboj et al., 2013; Attwood and Munafo, 2014).

Six facial expressions of emotion are generally accepted as being universally recognized: happiness, sadness, fear, anger, surprise and disgust; some researchers would also include contempt among the set of core expressions (Darwin, 1872; Ekman and Friesen, 1971; Waller et al., 2008). Several studies have suggested that alcohol affects the perception of some or all of these facial expressions of emotion, although the results have not always been consistent. Early findings indicated that the acute consumption of alcohol at a dose considered to be moderate $(0.5-0.55 \mathrm{~g} / \mathrm{kg}$; Tucker and Vuchinich, 1983) impairs the processing of facial expressions of emotion in general, and that higher doses might disrupt the recognition of anger in particular (Borrill et al., 1987; more recently supported by Stevens et al., 2008). An 
indiscriminate reduction in the recognition of facial expressions of emotion after alcohol (approximately $0.8 \mathrm{~g} / \mathrm{kg}$ ) was also reported by Tcherkassof et al. (2011), who used an atypical set of naturally-recorded expressions presented dynamically. Congruently, Craig et al. (2009) found that $0.4 \mathrm{~g} / \mathrm{kg}$ alcohol significantly raised recognition thresholds across facial expressions of emotion, producing a particularly marked effect on the recognition of sadness. A similar impairment in the recognition of sadness after $0.4 \mathrm{~g} / \mathrm{kg}$ was reported by Attwood $e t$ al. (2009a), albeit for male participants only; no other effects were found. Altered sensitivity to expressions of sadness may also be apparent in the findings of Kamboj et al. (2013): using dynamic expressions, they found an enhanced response bias to neutral expressions at $0.4 \mathrm{~g} / \mathrm{kg}$ alcohol, an effect that they attributed to participants tending to mislabel sad expressions as neutral.

In contrast, using another paradigm in which morphed images varied from unambiguously angry to unambiguously disgusted or from unambiguously angry to unambiguously happy, Attwood et al. (2009b) found that $0.4 \mathrm{~g} / \mathrm{kg}$ alcohol produced only a selective tendency to label disgust as anger, and only when the stimuli were male. The limited effects of $0.4 \mathrm{~g} / \mathrm{kg}$ on anger and happiness were supported by Walter et al. (2011), who found no effects of alcohol on the recognition of these expressions. However, other work has highlighted selective effects on emotional expressions other than sadness or anger: Kano et al. (2003) suggested that alcohol may specifically enhance the recognition of happy expressions, but only at a low dose $(0.14 \mathrm{~g} / \mathrm{kg})$.

Clearly, previous studies have not been entirely consistent. A tentative synthesis of the findings might be that alcohol at higher doses (over $0.4 \mathrm{~g} / \mathrm{kg}$ ) tends to produce a generalized reduction in identification accuracy for facial expressions of emotion, perhaps with a particular impact on anger recognition, whereas lower doses sometimes cause impairment 
across expressions (depending on the paradigm adopted) and may selectively impair the recognition of sadness.

Although a number of different procedures have been employed to assess the effects of alcohol on the recognition of emotional expression, probably contributing to the diversity of outcomes, none have explicitly contrasted expressions presented at brief and different durations. Some facial expressions of emotion occur very briefly (lasting between 40-200 ms) and are often referred to as "microexpressions" to distinguish them from longer-duration expressions (Ekman, 1992; Haggard and Isaacs, 1966). Unlike the latter, microexpressions of emotion are difficult to generate - or to inhibit - voluntarily (Frank and Ekman, 1997). The recognition of microexpressions is typically worse for those expressions of emotion which are less frequently encountered, such as fear and disgust (Porter and ten Brinke, 2008; Shen et al., 2002).

The aim of the present study was to examine how alcohol affects a person's ability to recognise microexpessions of emotions at doses within the range typical of social gatherings. For comparison, we also tested alcohol's effects on the recognition of facial expressions of emotion presented for longer than the microexpression duration. Two doses of alcohol were tested since the dose-response relationship for alcohol may not be linear (e.g. Carpenter and Ross, 1965; Borrill et al., 1987; Maylor et al., 1987; Lloyd and Rogers, 1997) and to encompass the range commonly adopted in earlier studies. Based on previous research it was predicted that alcohol would diminish recognition accuracy in general and increase the time needed for accurate responses, perhaps disrupting the recognition of sadness in particular. It might also be predicted that the impairment of executive function associated with alcohol would lead to particular difficulties in recognising microexpressions of emotion in 
comparison with the longer expressions of emotion which allow for longer deliberation and response choice.

\section{METHODS}

\section{Participants}

The sample comprised 18 undergraduate students from Kingston University out of a total of 21 tested ( 17 females, 4 males; mean age $=23$ years, $S D=7$ years $) ; 3$ participants in the original sample of 18 produced outlier values for baseline accuracy and were removed (none of their data are shown here) and replaced by 3 new participants. All participants were recruited via opportunity sampling and were offered course credits or a $£ 15$ voucher in return for participation. All had normal or corrected-to-normal vision, and none had a history of alcohol-related problems, as determined by the Michigan Alcohol Screening Test (Selzer et al., 1971). They were social drinkers (minimum of 12/14 UK units consumed weekly by women/men, respectively; maximum 30 units/week; 1 UK unit $=8 \mathrm{~g}$ alcohol) who drank an equivalent number of units to the highest dose given here in a single session at least once every two weeks. They were in good health (by self-report) and not taking any medication (exception: the contraceptive pill), they had not experienced any unusual adverse reactions to alcohol, and they were not pregnant or trying to become pregnant (by self-report). Participants gave written informed consent after being told that the purpose of the experiment was to test the effects of alcohol on emotion recognition. The research protocol was approved by the Kingston University Faculty of Arts and Social Sciences Ethics Committee, and the study was conducted according to the ethical standards of the British Psychological Society and the Declaration of Helsinki 1964.

\section{Materials}




\section{Drinks}

Drink formulations were derived from Terry et al. (2009). The "high dose" of alcohol was 0.6 $\mathrm{g} / \mathrm{kg}$ (males) and $0.52 \mathrm{~g} / \mathrm{kg}$ (females), and the "low dose" was one-third of the high dose, i.e. $0.2 \mathrm{~g} / \mathrm{kg}$ (males) and $0.17 \mathrm{~g} / \mathrm{kg}$ (females). The alcohol drinks comprised Waitrose vodka (37\% alcohol-by-volume) plus diet Schweppes Indian tonic water to a total beverage volume of $240 \mathrm{ml}$, plus $4 \mathrm{ml}$ Angostura Bitters. The placebo drink replaced vodka with equivalent tonic water, and 3-4 drops of vodka were floated on the drink surface and around the rim of the glass to mask olfactory cues; 4 ml Angostura Bitters was added.

\section{Stimulus materials}

The stimuli consisted of facial expressions of emotion (anger, contempt, disgust, fear, happiness, and sadness) and correspondent neutral faces produced by 12 male actors. The stimuli had been used extensively in previous studies in the laboratory (primarily using microexpression durations between 100-200 ms) in the absence of alcohol, and accuracy for identifying the emotions was stable and replicable. We omitted 'surprise' from the set of expressions because our previous studies had shown that recognition accuracy for surprise was as high as for joy/happiness at all durations and across participants, whereas the accuracy for the other emotions tended to vary with duration and/or participant. Also no previous alcohol study had shown any indication of an effect on surprise recognition. Instead, we substituted a facial expression that has not been studied extensively before in studies of alcohol's effects: 'contempt'. Contempt is often considered one of the primary emotions (e.g. Ekman and Heider, 1988). The images were obtained from the JACFEE/JACneut slide set (Matsumoto and Ekman, 1988), which was based on the "Pictures of Facial Affect" (POFA) database by Ekman. Six avatars of Caucasian-like male faces were created using the program FaceGen Modeller 3.5. The hair and external contours of some POFA faces were erased before they were overlaid on the avatars using Photoshop CS5. The final pairs of faces 
(neutral + emotion expression) were cropped to ensure that the facial expressions of emotion were central in the images. The images were then incorporated into a computerized presentation task using E-Prime software (Psychology Software Tools, Inc.). Examples of the images are presented in Figure 1.

\section{-- FIGURE 1 APPROXIMATELY HERE --}

\section{Procedure}

A repeated-measures design was adopted: participants were each tested three times: once with each of the three different drinks (placebo, "low" and "high" doses of alcohol). Drink order was counterbalanced across the 18 participants ( 3 blocks of 6 possible drink orders). Testing of a given participant occurred over 3 separate days, with at least $48 \mathrm{hrs}$ between test sessions. Participants were asked to abstain from alcohol and other drugs from at least the night before each session, and to avoid all food within the 2 hours preceding a scheduled test session. A breathalyser reading (Lion Alcometers) was taken to confirm recent abstinence from alcohol (all participants tested at zero). They were then weighed and they waited in a room adjoining the laboratory while the drink for that session was prepared. The drink was divided into three small cups of equal size and participants were asked to drink each cup steadily for 5 min per cup. After consumption, participants were asked to wait 20 min before taking the computer-based test, during which period they could read a magazine provided by the experimenter. A second breathalyser test was conducted, and then the computerized emotion-recognition test began immediately afterwards.

The stimulus viewing angle was approximately $6 \times 5$ degrees at $65 \mathrm{~cm}$ from the centre of the monitor. A practice test phase comprised six trials. In each trial, a sequence of 3 avatars was presented: a neutral expression (1000 ms), followed by an emotion expression (300 ms), and back to the neutral expression (1000 ms). Once the sequence finished, a screen containing six buttons - each with the name of one of the six emotions - appeared, and the 
participant had to choose the name of the emotion which had been presented between the two neutral ones with a mouse click. The actual test started soon after the practice test. The tests were identical, except for the duration of the microexpressions, which were either $200 \mathrm{~ms}$ or $400 \mathrm{~ms}$, always preceded and followed by the correspondent $1000 \mathrm{~ms}$ neutral expressions. Feedback was provided after each trial. There were 24 trials per session $(6$ emotions x 2 repetitions/emotion $\mathrm{x} 2$ durations). The presentation order of the microexpressions was randomized. Within-participant tests occurred at the same time of day; the time of testing varied between participants but was always between 14:00 and 17:00. Participants were asked to remain on campus for 2 hours after completing the test, and they were advised not to drive, cycle or engage in any hazardous activity for the rest of the day.

A pilot study (no drinks) was conducted with 34 participants to test whether the position of the buttons on the screen (left or right) affected response accuracy. Since no significant differences were observed, the position of the buttons showing the names of the emotions was kept the same in all trials in the present study.

\section{Data analyses}

Analyses were by repeated-measures analysis of variance (ANOVA), with factors Emotion (6 levels: anger, contempt, disgust, fear, happiness, sadness) and Drink (3 levels: placebo, lowdose, high-dose). For simplicity, the two durations of presentation (microexpressions at 200 ms, longer expressions at $400 \mathrm{~ms}$ ) were analysed separately. The dependent variables were accuracy of emotion identification (percentage correct) and reaction time (milliseconds, ms). Responses shorter than $0.1 \mathrm{~s}$ or longer than $10 \mathrm{~s}$ were eliminated from the data analyses as errors. The confidence intervals (95\% CI) are given in parentheses where appropriate. Greenhouse-Geisser adjustments to the degrees of freedom were performed when sphericity could not be assumed (Mauchly's sphericity test). Partial eta-squared ( $\left.\eta p^{2}\right)$ was used to refer to effect size. Pairwise comparisons were carried out using Bonferroni adjustments, and post 
hoc comparisons using paired $t$-tests were used where significant interactions were identified. The software $\mathrm{G}^{*}$ Power was used to calculate the Cohen's $d$ for the $t$-tests, which (as a rule of thumb) is classified as small (up to 0.2 ), medium $(0.2<\mathrm{x} \leq 0.5$ ), or large (up to 0.8 ).

\section{RESULTS}

\section{Brief duration presentations: microexpressions of emotion (200 $\mathrm{ms})$}

Accuracy. Repeated measures ANOVA revealed a significant main effect of Emotion on identification accuracy $\left(F(3.08,52.42)=14.02, p<.001, \eta p^{2}=.45\right)$ and an interaction between Emotion and Drink (placebo, low or high dose: $F(5.04,85.61)=3.27, p=.001, \eta p^{2}$ $=.16)$. However, there was no main effect of Drink on accuracy $(F(2,34)=2.01, p=.15$, $\left.\eta p^{2}=.11\right)$. All mean accuracy scores and their respective $95 \%$ confidence intervals are given in Table 1. A comparison of the overall identification accuracies for the different microexpressions, collapsing across alcohol conditions to yield simple effects, revealed that the recognition of disgust was significantly worse than the recognition of contempt $(p<$ $.001)$, fear $(p<.001)$, happiness $(p<.001)$, and sadness $(p=.005)$. The recognition of anger differed significantly only from the recognition of happiness $(p=.02)$.

The high dose of alcohol significantly affected the recognition accuracy of certain microexpressions, but the effects of the low dose were indistinguishable from those of the placebo across all microexpressions. Paired-samples $t$-tests showed that identification accuracy for contempt was raised from $85 \%$ with placebo to $99 \%$ with the high dose of alcohol $(t(17)=-2.15, p=.045$, Cohen's $d=0.51)$, which was also significantly higher than with the low dose of alcohol $(90 \% ; t(17)=2.38, p=.03, d=0.61)$. The same pattern of effect was observed for disgust: identification accuracy increased from $61 \%$ in the absence of alcohol to $89 \%$ at the high dose $(t(17)=-2.70, p=.015, d=0.65)$; again, the high dose scores 
were also significantly greater than the low dose scores $(59 \%, t(17)=3.40, p=.003, d=$ 0.81). There was a tendency for alcohol to reduce identification accuracy for anger, but the effect was not statistically reliable. The significant effects of alcohol on disgust and contempt are illustrated in Figure 2, alongside mean accuracies for the identification of anger.

\section{-- TABLE 1 AND FIGURE 2 APPROXIMATELY HERE -}

Reaction time. There was a significant difference between response latencies for the different microexpressions $\left(F(3.43,58.25)=4.44, p=.005, \eta p^{2}=.21\right)$, but no reliable interaction between Emotion and Drink and no main effect of Drink. Although the placebo and alcohol conditions did not differ significantly from each other in terms of mean RT, there was a tendency towards shorter RT in the placebo condition in comparison with the highalcohol condition across microexpressions (Table 2).

\section{-- TABLE 2 APPROXIMATELY HERE -}

\section{Longer presentations of facial expressions of emotion (400 ms)}

Accuracy. Overall accuracy improved when the duration of the emotional expression was doubled to $400 \mathrm{~ms}$. There was a significant effect of Emotion on identification accuracy $\left(F(2.81,47.79)=8.79, p<.0001, \eta p^{2}=.34\right)$ and a significant interaction between Emotion and Drink $\left(F(10,170)=2.91, p=.002, \eta^{2}=.15\right)$. The main effect of Drink approached significance $\left(F(2,34)=3.21, p=.053, \eta p^{2}=.16\right)$. When averaged across all Drink conditions, the accuracy for the recognition of anger was significantly worse than for the recognition of happiness $(p=.002)$ and sadness $(p=.01)$, whereas the recognition of disgust was worse than the recognition of fear $(p=.015)$, happiness $(p=.002)$ and sadness $(p=.003)$. The recognition of contempt was only worse than the recognition of happiness $(p=.002)$.

Paired-samples $t$ tests revealed a profile of effects of alcohol with longer emotional expressions that was similar to those produced by the microexpressions: recognition accuracy 
for contempt was significantly better after the high-dose of alcohol (97\%) than after placebo $(77 \% ; t(17)=-3.10, p=.007, d=0.71)$, as was the recognition accuracy for disgust $(72 \%$ after placebo and 99\% after the high dose: $t(17)=-3.12, p=.006, d=0.76)$. However, for both contempt and disgust, recognition accuracy after the low dose of alcohol did not differ significantly from accuracy after the high dose (for each: $p>0.05$ ). Figure 2 shows the effects of alcohol on disgust, contempt and anger specifically.

Reaction time. There was a significant difference between the reaction times for recognition of the different emotions $\left(F(2.80,47.60)=5.16, p=.004, \eta p^{2}=.23\right)$, but there was no significant main effect of Drink $\left(F(1.51,21.68)=1.23, p=.30, \eta p^{2}=.07\right)$ and no interaction between Emotion and Drink $\left(F(3.97,67.42)=1.24, p=.30, \eta p^{2}=.07\right)$. Paired comparisons between emotions only revealed significantly slower RT to recognize anger in comparison with fear $(p=.02)$ and happiness $(p=.012)$; see Table 2.

\section{DISCUSSION}

This study investigated the effect of alcohol on the recognition of brief-duration facial expressions of emotion (microexpressions, $200 \mathrm{~ms}$ ), as well as on longer-duration expressions (400 ms). The presentation of microexpressions produced a significant interaction between the emotion depicted and the amount of alcohol consumed. The recognition of disgust was low in comparison with the recognition of other emotions, but the high dose of alcohol ( 0.6 or $0.57 \mathrm{~g} / \mathrm{kg}$ for men and women respectively) led to an increase in recognition accuracy for disgust relative to the other two conditions. A similar profile of effects was observed after presentation of the facial expression of contempt. Presenting the expressions of emotion at the longer duration of $400 \mathrm{~ms}$ replicated these effects: a similar, significant interaction occurred, whereby the higher dose of alcohol selectively improved the recognition of disgust and contempt. The similar profiles of effects across the two 
presentation durations (supported by the absence of a main effect of duration in ANOVA combining the two durations, results not shown) implies that the same underlying mechanisms contribute to emotion recognition at both durations, and that microexpressions are not differentially sensitive to alcohol's effects (e.g. via greater vulnerability to alcohol's effects on executive function). In contrast to its effects on accuracy, alcohol did not significantly affect the response latencies to any of the stimuli at either presentation duration.

Some previous studies have reported an impairment of anger recognition after alcohol (Borrill et al., 1987; Stevens et al., 2008); in the present study, the recognition of microexpressions of anger was lower after both doses of alcohol relative to placebo, but not significantly, and there was no indication of such an effect at the longer stimulus duration. Similarly, we did not detect any impairment in the recognition of sadness, unlike some previous studies (Attwood et al., 2009a; Craig et al., 2009).

The effect of the higher dose of alcohol to improve recognition accuracy for expressions of disgust and contempt was surprising and has possible implications for social behaviour. It might be argued that an increased sensitivity to expressions of contempt (in particular) after the consumption of alcohol in a social context may have deleterious consequences, in that it could elevate the risk of alcohol-associated aggression in reaction to the provocative social cue. The implications of the results are consistent with others that have raised concerns about how alcohol's effects on face processing might be relevant to its effects on aggression and violence (e.g. Attwood et al., 2009a; 2009b; Attwood and Munafo, 2014). Although these effects on contempt and disgust were not anticipated, it is important to note that these were not disparate post hoc outcomes: the reliability of the phenomena is supported by internal replication at both presentation durations for both expressions, and moreover the enhancements were apparent by post hoc comparison with both placebo and low-dose conditions for microexpressions (at the longer duration of presentation, only high-dose versus 
placebo comparisons were significant). A general alcohol-induced bias towards clicking a negative response could not easily account for the full pattern of results, since alcohol tended to reduce recognition accuracy for 'anger' (a near-significant decline in the microexpression condition); in addition, collapsing across alcohol conditions, the recognition of 'anger' was significantly worse than for the recognition of happiness at both of the presentation durations (and also worse than for sadness at the longer presentation duration). Although it is difficult to identify analogous effects in previous studies of alcohol's effects on emotion recognition, there may be at least one precedent: Borrill et al (1987) showed a subtle effect of alcohol on the identification of disgust/contempt (different from the emotions tested in the present study, but interestingly a composite of the two emotions highlighted here): their results hinted at improved recognition accuracy after approximately $0.3 \mathrm{~g} / \mathrm{kg}$ alcohol relative to both placebo and a higher dose of alcohol (approximately $0.74 \mathrm{~g} / \mathrm{kg}$ ), albeit in males only. In contrast, Attwood et al. (2009a) found that alcohol at $0.4 \mathrm{~g} / \mathrm{kg}$ increased the likelihood of categorizing morphed images as angry rather than disgusted (but only for male stimuli), and Kamboj et al. (2013), using a different dynamic morphing procedure, found no reliable effect of alcohol on disgust either at 0.4 or $0.8 \mathrm{~g} / \mathrm{kg}$. Other studies have not tested or reported the effects of alcohol on the recognition of disgust (Tucker and Vuchinich, 1983; Kano et al., 2003; Stephens et al., 2008; Attwood et al., 2009b; Craig et al., 2009; Tcherkassof et al., 2011; Walter et al., 2011) and there are no reports of alcohol's effects on the recognition of contempt (except in the case of the hybrid stimuli of Borrill et al., 1987).

On the other hand, there is another prior report of drug-associated enhancement of disgust recognition that does not involve alcohol administration. Martin et al. (2006) reported a similar degree of improved performance in opiate-maintained participants (primarily receiving methadone) relative to opiate-abstinent participants. Given that opiate drugs are strongly immunosuppressant (e.g. Roy and Loh, 1996; Vallejo et al., 2004), the intriguing 
suggestion is made by Curtis et al. (2011) that disgust sensitivity may be heightened by circulating opiates as a protective reaction against exposure to infection when the immune system is compromised. State changes in disgust sensitivity during immunosuppression have also been proposed by others (e.g. Fessler and Navarrete, 2003; Stevenson et al., 2009). There is evidence that alcohol administered at moderate doses can disrupt immune function in ways that might increase vulnerability to infection (Szabo et al., 1999). That being the case, it might be argued that the impairment of immune function following the consumption of occasional moderate-to-high doses of alcohol could increase sensitivity to signals of disgust as a protective response against infection, even though long-term alcohol abuse is associated with changes in immune function that can increase infection susceptibility (e.g. Cook, 1998; Friedman et al. 2003). To our knowledge, nobody to date has suggested that alcohol can produce behavioural changes that are consistent with elevated sensitivity to stimuli indicating infection or contagion, let alone tied such responses directly to alcohol-induced changes in immune function. In fact, several studies that have tested the recognition of facial expressions of emotion in alcoholics have shown consistently that alcoholics are significantly less accurate than social drinkers at identifying all emotional expressions, including disgust (e.g. Philippot et al., 1999; Frigerio et al., 2002; Townshend and Duka, 2003). Of course, alcohol abuse can cause multiple systemic changes, which may be permanent, and these changes might negate any influence of disrupted immune function on the recognition of disgust. Hence the data from alcoholics may not necessarily rule out a link between altered immune function and disgust recognition following more modest alcohol consumption by social drinkers.

Nevertheless, an explanation of the present outcomes in terms of alcohol intake providing a signal of impaired immune function would not account for the enhanced recognition of contempt identified here. Perhaps a more compelling explanation for the effects of alcohol on 
both categories of emotion is that contextual priming may operate to predispose a bias towards the recognition of disgust and contempt in the specific circumstances of the current experiment. Studies of alcohol's effects on emotion recognition differ substantially in terms of sample composition, test circumstances, the nature of the communication between participant and experimenter, and the motivations and incentives for participation. These factors all have the potential to engender particular priming effects, even before taking account of the wide variety of stimuli that have been adopted and the different modes of presentation employed. For example, Martin et al. (2006) argued that hypersensitivity to expressions of disgust by opiate-maintained participants might reflect their prior exposure, over a sustained period, to other people's negative evaluations of their status as opiate abusers. Feelings of shame, reinforced by social disapproval, might therefore favour the recognition of expressions of disgust and contempt, since these expressions - in which the gaze is directed at the participant in the present stimulus set - could be construed as conveying negative judgments about the participant. The issue then arises: why should the context of the current experiment prime such specific reactions whereas previous, similar experiments have not shown analogous effects? First, as noted, Borrill et al. (1987) produced analogous findings, so the effect is not isolated. Secondly, other studies have not tested contempt and they have not always tested disgust; for those studies in which disgust has been tested, other procedures have been adopted. A social context influence might have been exacerbated in the current study by having a predominantly female sample being tested by a male experimenter, and by using exclusively male stimuli. Previous studies have found gender effects specific to particular face genders, outcomes that have not been predicted or fully explained in previous studies. Hence it would be useful to test more males for comparison with females, and perhaps to manipulate the gender of the stimuli. Although the number of participants was modest, it was not inconsistent with other studies that have used 
less powerful between-groups designs (e.g. Borrill et al., 1987; Martin et al., 2006;

Tcherkassof et al., 2011; Kamboj et al., 2013) or repeated-measures designs (e.g. Kano et al., 2003). Furthermore, we report effect sizes - unlike most previous studies cited here - which are indicative of large-to-medium effects for the critical comparisons, suggesting that power is adequate. Finally, a procedure that allows for threshold derivation by using "morphed" stimuli or by extending the stimulus set with a larger range of expression "intensities" might allow for more nuanced comparison between emotions, in particular by eliminating ceiling effects for emotions like "happiness". Different procedures and stimulus sets are likely to yield different baseline accuracy rates and thresholds, with consequences for detecting alcohol-induced increases or decreases in recognition accuracy. This problem is not necessarily overcome just by increasing the range of intensities presented or the numbers of presentations. The full facial expressions used here were not all equally well-recognised and, except for happiness, the accuracy for the expressions was not at ceiling. The lower baseline rates for recognising disgust and contempt provide greater potential for detecting a facilitatory effect of alcohol, but the fact that anger recognition in particular tended to decline after alcohol suggests that the alcohol effect was not one of indiscriminate facilitation. Clearly, the current procedure was sensitive to alcohol's effects, as have been other procedures using limited sets of stimuli (e.g. Tucker et al., 1983; Borrill et al., 1987; Kano et al., 2003).

As already mentioned, it is difficult to compare clearly and objectively the several studies that have tested the effects of alcohol on emotion recognition, not only because of the relatively wide range of doses used (from $0.14-0.80 \mathrm{~g} / \mathrm{kg}$ ), but also due to the different experimental paradigms employed. Previous studies have not produced consistent outcomes themselves in relation to other emotional expressions (e.g. anger, sadness, happiness). Parametric manipulation of variables within the same basic methodology, rather than 
comparisons across widely differing procedures, will help to clarify the key influences on performance. The current study suggests further that manipulating the social context of testing and systematically exploring the demand characteristics inherent in these procedures might provide a way forward to better understand alcohol's effects on emotion recognition. 


\section{REFERENCES}

Attwood AS, Ataya AF, Benton CP, Penton-Voak IS, Munafo MR. 2009a. Effects of alcohol consumption and alcohol expectancy on the categorisation of perceptual cues of emotional expression. Psychopharmacol 204: 327-334.

Attwood AS, Munafo MR. 2014. Effects of acute alcohol consumption and processing of emotion in faces: implications for understanding alcohol-related aggression. $J$ Psychopharmacol 28: 23-30.

Attwood AS, Ohlson C, Benton CP, Penton-Voak IS, Munafo MR. 2009b. Effects of acute alcohol consumption on processing of perceptual cues of emotional expression. $J$ Psychopharmacol 23: 23-30.

Blair RJ. 2003. Facial expressions their communicatory functions and neurocognitive substrates. Philos Trans R Soc Lond B 358: 561-572.

Borrill JA, Rosen BK, Summerfield AB. 1987. The influence of alcohol on judgment of facial expression of emotion. Br J Med Psychol 60: 71-77.

Carpenter JA, Ross BM. 1965. Effect of alcohol on short-term memory. Quart J Stud Alcohol 26: $561-579$.

Craig LC, Attwood AS, Benton CP, Penton-Voak IS, Munafo MR. 2009. Effects of acute alcohol consumption and alcohol expectancy on the processing of perceptual cues of emotional expression. J Psychopharmacol 23: 258-265. 
Cooper ML, Frone MR, Russell M, Mudar P. 1995. Drinking to regulate positive and negative emotions: a motivational model of alcohol use. J Pers Soc Psychol 69: 990-1005.

Curtin, JJ, Patrick CJ, Lang, AR, Cacioppo JT, Birbaumer N. 2001. Alcohol affects emotion through cognition. Psychol Sci 12: 527-531.

Curtis V, de Barra M, Aunger R.2011. Disgust as an adaptive system for disease avoidance behaviour. Phil Trans $R$ Soc B 366: 389-401

Darwin CR. 1872. The expression of the emotions in man and animals (1st ed.). London: John Murray.

Donohue KF, Curtin, JJ, Patrick CJ, Lang AR. 2007. Intoxication level and emotional response. Emotion 7: 103-112

Eckhardt CI. 2007. Effects of alcohol intoxication on anger experience and expression among partner assaultive men. J Consult Clin Psychol 75: 61-71

Ekman P. 1992. Facial expressions of emotion: new findings, new questions. Psychol Science 3: $34-38$.

Ekman P, Friesen WV. 1971. Constants across cultures in the face and emotion. J Pers Soc Psychol 17: 124-129.

Ekman P, Heider KG. 1988. The universality of a contempt expression: A replication. Motivation and Emotion 12: 303-308 
Fessler DMT, Navarrete CD. 2003. Domain-specific variation in disgust sensitivity across the menstrual cycle. Evol Hum Behav 24: 406-417.

Frank MG, Ekman P. 1997. The ability to detect deceit generalizes across different types of high-stake lies. J Pers Soc Psychol 72: 1429-1439.

Frigerio E, Burt DM, Montagne B, Murray LK, Perrett DI. 2002. Facial affect perception in alcoholics. Psychiatry Res 113: 161-171.

Giancola PR. 2000. Executive functioning: a conceptual framework for alcohol-related aggression. Exp Clin Psychopharmacol 8: 576-597.

Giancola PR. 2004. Executive functioning and alcohol-related aggression. J Abnorm Psychol 113: 541-555.

Haggard EA, Isaacs KS. 1966. Micro-momentary facial expressions as indicators of ego mechanisms in psychotherapy. In L. A. Gottschalk \& A. H. Auerbach (Eds.), Methods of Research in Psychotherapy (pp. 154-165). New York: Appleton-Century-Crofts.

Kamboj SK, Joye A, Bisby JA, Das RK, Platt B, Curran HV. 2013. Processing of facial affect in social drinkers: a dose-response study of alcohol using dynamic emotion expressions, Psychopharmacol 227: 31-39.

Kano M, Gyoba J, Kamachi M, Mochizuki H, Hongo M, Yanai K. 2003. Low doses of alcohol have a selective effect on the recognition of happy facial expressions. Hum Psychopharmacol - ClinExp 18: 131-139. 
Lloyd HM, Rogers PJ.1997. Mood and cognitive performance improved by a small amount of alcohol given with a lunchtime meal. Behav Pharmacol 8:188-195.

Martin L, Clair J, Davis P, O’Ryan D, Hoshi R, Curran HV. 2006. Enhanced recognition of facial expressions of disgust in opiate users receiving maintenance treatment. Addiction 101: $1598-1605$.

Matsumoto D, Ekman, P. 1988. Japanese and Canadian facial expression (JACFEE) and neutral faces (JACneut). San Francisco: San Francisco State University.

Maylor EA, Rabbitt PMA, Sahgal A, Wright C. 1987. Effects of alcohol on speed and accuracy in choice reaction time and visual search. Acta Psychologica 65:147-163.

Philippot P, Kornreich C, Blairy S, Baert I, Den Dulk A, Le Bon O, Streel, E, Hess U, Pelc I, Verbanck P. 1999. Alcoholics' deficits in the decoding of emotional facial expression. Alc Clin Exp Res 23: 1031-1038.

Porter S, ten Brinke L 2008. Reading between the lies: Identifying concealed and falsified emotions in universal facial expressions. Psychol Sci 19: 508-514.

Roy S, Loh HH. 1996. Effects of opioids on the immune system. Neurochem Res 21: 13751386.

Sayette MA, Creswell KG, Dimoff JD, Fairbairn CE, Cohn JF, Heckman BW, Bryan W, Kirchner TR, Levine, JM, Moreland, RL. 2012. Alcohol and group formation: a multimodal investigation of the effects of alcohol on emotion and social bonding. Psychol Sci 23: 869878. 
Selzer ML, Vanosdall FE, Chapman M. 1971. Alcoholism in a problem driver group: Field trial of Michigan Alcoholism Screening Test (MAST). J Safety Res 3: 176-181.

Shen X, Wu Q, Fu X. 2012. Effects of the duration of expressions on the recognition of microexpressions. J Zhejiang Univ-Sci B (Biomed Biotechnol) 13: 221-230.

Stappenbeck CA, Fromme K. 2014. The effects of alcohol, emotion regulation, and emotional arousal on the dating aggression intentions of men and women. Psychol Addict Behav 28: 10-19.

Stevens S, Gerlach AL, Rist F. 2008. Effects of alcohol on ratings of emotional facial expressions in social phobics. J Anx Dis 22: 940-948.

Stevenson RJ, Case TI, Oaten MJ. 2009. Frequency and recency of infection and their relationship with disgust and contamination sensitivity. Evol Hum Behav 30: 363-368.

Szabo G. 1999. Consequences of alcohol consumption on host defence. Alc Alc 34: 830-841.

Tcherkassof A, Mandran N, Dubois M, Begue L. 2011. The effects of acute alcohol drinking on the judgement of spontaneous and dynamic facial expressions of emotion. Psychologie Francaise 56: 189-202.

Terry P, Doumas M, Desai RI, Wing AM. 2009. Dissociations between motor timing, motor coordination, and time perception after the administration of alcohol or caffeine.

Psychopharmacol 202: 719-729.

Townshend JM, Duka T. 2003. Mixed emotions: alcoholics' impairments in the recognition of specific emotional facial expressions. Neuropsychologia 41: 773-782. 
Tucker JA, Vuchinich RE. 1983. An information processing analysis of effects of alcohol on perceptions of facial emotions. Psychopharmacol 79: 215-219.

Vallejo R, de Leon-Casasola O, Benyamin R. 2004. Opioid therapy and immunosuppression: a review. Am J Ther 11: 354-365.

Waller BM, Cray JJ, Burrows AM. 2008. Selection for universal facial emotion. Emotion 8: 435-439.

Walter NT, Mutic S, Markett S, Montag C, Klein AM, Reuter M. 2011. The influence of alcohol intake and alcohol expectations on the recognition of emotions. Alc Alcohol 46: 680685.

Westmaas J, Moeller S, Woicik PB. 2007. Validation of a measure of college students intoxicated behaviors: associations with alcohol outcome expectancies, drinking motives, and personality. J Am Coll Health 55: 227-237.

Williams F, Hasking P. 2010. Emotion regulation, coping and alcohol use as moderators in the relationship between non-suicidal self-injury and psychological distress. Prevention Sci 11: $33-41$. 


\section{FIGURES}

Figure 1. A: Versions of two of the facial expressions of emotion used in the study (upper row: neutral - happiness - neutral; lower row: neutral - disgust- neutral). B: Timeline of a typical trial; the example shows presentation of a microexpression (200 ms) of happiness. The duration of the emotional image was either $200 \mathrm{~ms}$ or $400 \mathrm{~ms}$ (only $200 \mathrm{~ms}$ is shown here). All trials were randomly interleaved.

Figure 2: Response accuracy (mean percentage correct) for recognition of facial expressions of disgust, contempt and anger, each presented for either $200 \mathrm{~ms}$ (upper graph) or $400 \mathrm{~ms}$ (lower graph). Bars represent SEM; asterisk $=\mathrm{p}<0.05$ for the comparison indicated. $\mathrm{N}=18$ at all points in a repeated measures design. 
Figure 1.

$1000 \mathrm{msec} 200 \mathrm{msec} \quad 1000 \mathrm{msec}$
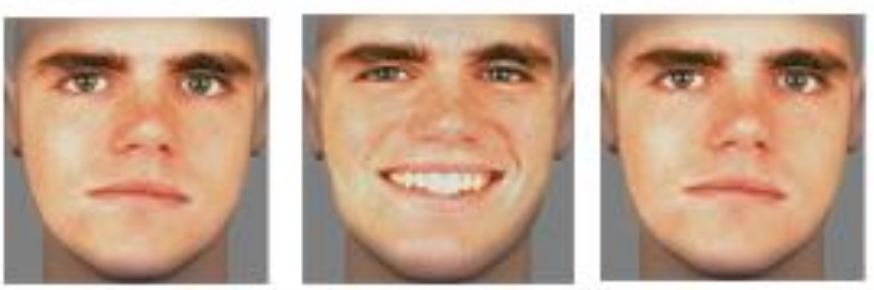

HAPPINESS
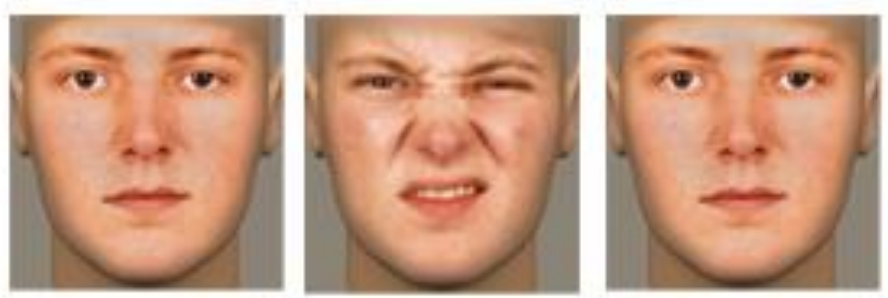

A

DISGUST

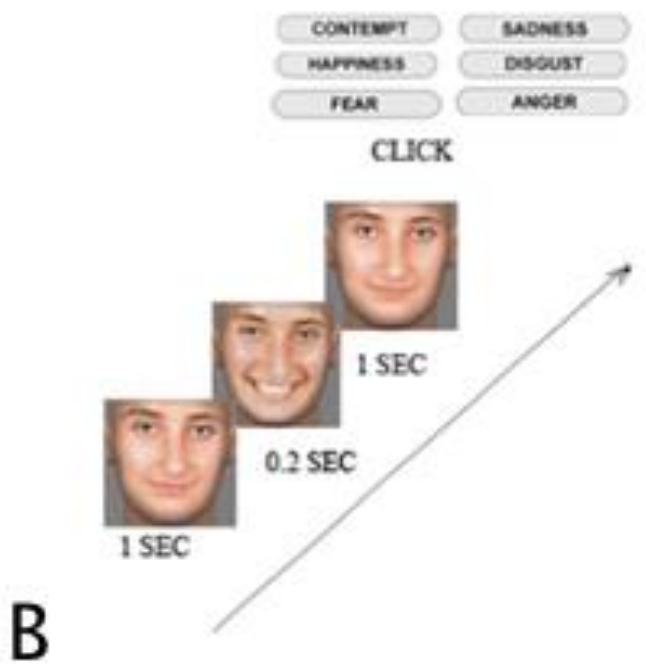


Figure 2
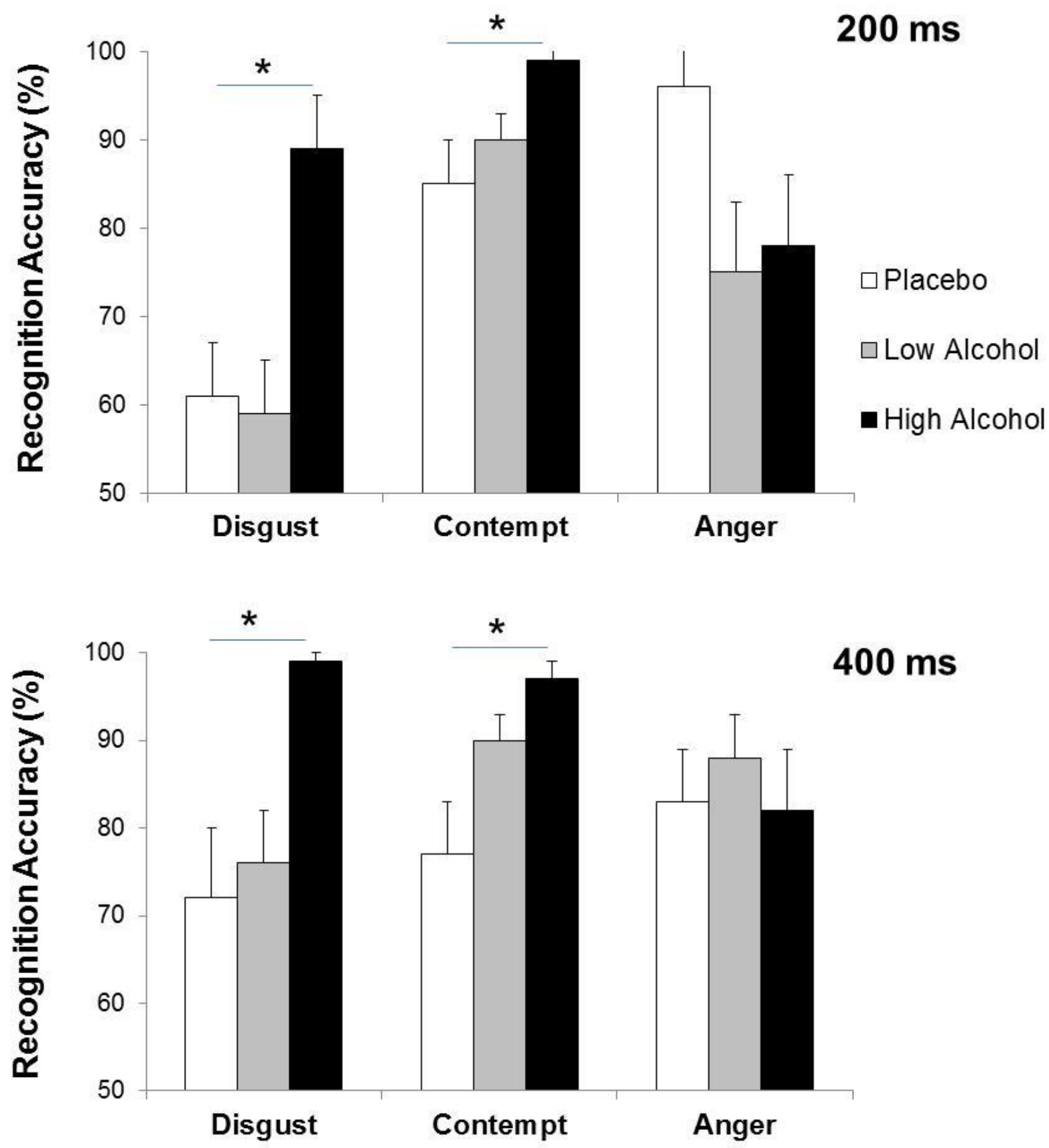
Table 1. Mean percentage accuracy and mean reaction time (ms) plus 95\% confidence intervals (CI) for the recognition of six facial expressions of emotion. The faces were displayed for either $200 \mathrm{~ms}$ (microexpression condition) or $400 \mathrm{~ms}$. The two presentation durations were randomly interleaved.

Duration 200 ms (mean, 95\% CI)

Duration 400 ms (mean, 95\% CI)

\section{Accuracy (\%)}

\begin{tabular}{lcclcll}
\hline & Placebo & Low Dose & High Dose & Placebo & Low Dose & High Dose \\
\hline Anger & $96[91,100]$ & $75[56,94]$ & $78[60,95]$ & $83[71,95]$ & $88[76,99]$ & $82[68,96]$ \\
\hline Contempt & $85[72,98]$ & $90[83,98]$ & $99[96,100]$ & $77[64,90]$ & $90[84,97]$ & $97[93,100]$ \\
\hline Disgust & $61[47,77]$ & $59[45,74]$ & $89[78,100]$ & $72[55,89]$ & $76[61,90]$ & $99[96,100]$ \\
\hline Fear & $97[93,100]$ & $89[76,100]$ & $94[88,100]$ & $99[96,100]$ & $92[80,100]$ & $97[93,100]$ \\
\hline Happiness & 100 & 100 & $96[89,100]$ & 100 & $97[93,100]$ & $97[93,100]$ \\
\hline Sadness & $94[85,99]$ & $85[71,96]$ & $90[80,95]$ & $99[96,100]$ & $96[89,100]$ & $97[93,100]$ \\
\hline
\end{tabular}


Table 2. Mean reaction time (ms) plus 95\% confidence intervals (CI) for the recognition of six facial expressions of emotion. The faces were displayed for either $200 \mathrm{~ms}$ (microexpression condition) or $400 \mathrm{~ms}$. The two presentation durations were randomly interleaved.

\section{Reaction Time (ms)}

\begin{tabular}{|c|c|c|c|c|c|c|}
\hline & Placebo & Low Dose & High Dose & Placebo & Low Dose & High Dose \\
\hline \multirow[t]{2}{*}{ Anger } & 774 & 980 & 1077 & 1090 & 912 & 1397 \\
\hline & {$[635,912]$} & {$[631,1308]$} & {$[748,1406]$} & {$[730,1450]$} & {$[660,1164]$} & {$[823,1970]$} \\
\hline \multirow[t]{2}{*}{ Contempt } & 716 & 971 & 965 & 838 & 852 & 802 \\
\hline & {$[581,851]$} & {$[680,1262]$} & {$[621,1309]$} & {$[653,1022]$} & {$[597,1106]$} & {$[569,1036]$} \\
\hline \multirow[t]{2}{*}{ Disgust } & 944 & 1064 & 1047 & 787 & 1005 & 1060 \\
\hline & {$[703,1185]$} & {$[823,1305]$} & {$[784,1311]$} & {$[614,960]$} & {$[782,1228]$} & {$[773,1346]$} \\
\hline \multirow[t]{2}{*}{ Fear } & 667 & 770 & 882 & 783 & 781 & 933 \\
\hline & {$[544,790]$} & {$[638,903]$} & {$[674,1091]$} & {$[621,945]$} & {$[599,964]$} & {$[671,1196]$} \\
\hline \multirow[t]{2}{*}{ Happiness } & 677 & 664 & 743 & 724 & 667 & 786 \\
\hline & {$[521,794]$} & {$[476,852]$} & {$[506,980]$} & {$[602,846]$} & {$[603,730]$} & {$[633,939]$} \\
\hline \multirow[t]{2}{*}{ Sadness } & 869 & 889 & 1037 & 853 & 694 & 845 \\
\hline & {$[624,1114]$} & {$[700,1078]$} & {$[577,1496]$} & {$[558,1149]$} & {$[587,801]$} & {$[655,1034]$} \\
\hline
\end{tabular}

